\title{
Extensive Intramyocardial Calcifications: Value of Multimodality Imaging
}

\author{
Fabiola Sozzi ${ }^{1}$, Laura Iacuzio ${ }^{2}$, Marco Schiavone $^{1}$, Filippo Civaia ${ }^{2}$, Stefano Carugo ${ }^{1}$, Franck \\ Levy $^{2}$, and Armand Eker ${ }^{2}$ \\ ${ }^{1}$ Fondazione IRCCS Ca' Granda Ospedale Maggiore Policlinico \\ ${ }^{2}$ Centre Cardio-Thoracique de Monaco
}

December 13, 2021

\begin{abstract}
Massive myocardial calcification is a very rare finding. Accurate identification and characterization may help the clinicians to determine the etiology and clinical significance. In this case, the diagnostic pathway excluded previous myocardial infarction, myocarditis and calcium-phosphate disorders. A possible dystrophic etiology was considered. There are no standardized imaging features available to classify specific subtypes of intramyocardial calcifications. The relative merits of cardiac computed tomography and magnetic resonance in providing complimentary diagnostic information for calcific myocardial lesions is shown.

Knowledge of the potential etiology and their imaging patterns are important to provide a concise and accurate differential diagnosis.
\end{abstract}

\section{Extensive Intramyocardial Calcifications:}

\section{Value of Multimodality Imaging}

Fabiola B Sozzi MD*, Laura Iacuzio MD, Marco Schiavone MD*, Filippo Civaia MD, Stefano Carugo MD*, Franck Levy MD, Armand Eker, MD

Cardiothoracic Centre CCM, Monaco, MC and

*Fondazione Ospedale Policlinico, IRCCS, Milan, IT

Running title: "A Rare Case of Intramyocardial Calcifications".

Word count: 250 words.

Keywords: Intramyocardial Calcifications. Cardiac CT. Cardiac MRI.

\section{Address for correspondence:}

Fabiola B Sozzi

Monaco Cardiothoracic Centre

Avenue d'Ostende 11

98004 Monaco

$\mathrm{Ph}+393295662258$

Email fabiola_sozzi@yahoo.it

\section{ABSTRACT}


Massive myocardial calcification is a very rare finding. Accurate identification and characterization may help the clinicians to determine the etiology and clinical significance. In this case, the diagnostic pathway excluded previous myocardial infarction, myocarditis and calcium-phosphate disorders. A possible dystrophic etiology was considered. There are no standardized imaging features available to classify specific subtypes of intramyocardial calcifications. The relative merit of cardiac computed tomography and magnetic resonance imaging in providing complimentary diagnostic information for calcific myocardial lesions is shown. Knowledge of the potential etiology and their imaging patterns are important to provide a concise and accurate differential diagnosis.

An 81-year-old woman, affected by Horton disease and with previous history of rheumatic fever, was hospitalized for acute chest pain. Physical examination and EKG were not significant. Chest X-Ray showed a diffuse hypodense lobulated area in the left ventricle (Figure 1: A). The echocardiogram revealed diffuse aortic and mitral calcifications with mild stenosis, marked septum and antero-lateral asymmetric hypertrophy, with extensive antero-lateral calcifications (Figure 1: B, Video 1). Non-contrast computed-tomography (CT) highlighted widespread amorphous confluent calcifications in the left ventricular wall, with septal sparing, extended to the mitral-aortic annulus and both coronary arteries (Figure 1: C-C1 showing VRT reconstruction and C2 MPR axial-view, Video 2). Cardiac magnetic resonance 3,0 T presented the following: septal hypertrophy, antero-lateral wall thickening with areas of intramyocardial signal-alteration, surrounded by normal myocardium matching the CT calcifications. Areas of low-signal were shown in multiple sequences: SSFP-cine in 4-chambers [4C] (Figure 2: D, Video 3) and in short-axis [SAX] (Figure 2: D1); T1-weightedspin-echo in 4C (Figure 2: E) and SAX (Figure 2: E1); STIR in 4C (Figure 2: F). T1-native-mapping in $4 \mathrm{C}$ focused on diffuse septum and lateral fibrosis with low-signal (550 ms, normal range: 1150-1250 ms) compatible with calcifications (Figure 2: G). After contrast-gadolinium injection, PSIR-sequence showed intramyocardial hypersignal in the lateral wall both in $4 \mathrm{C}$ and SAX, Figure 2: $\mathrm{H}-\mathrm{H} 1$ respectively). Coronary angiography documented significant disease that required revascularization. Extensive intramyocardial calcifications are extremely rare (1). In our case the diagnostic pathway excluded previous myocardial infarction, myocarditis and calcium-phosphate disorders. A possible dystrophic etiology was considered according also to the rheumatic disease. There are no standardized imaging features available to classify specific subtypes of intramyocardial calcifications (2). The combination of imaging pattern and potential etiology plays a key role in characterizing calcifications and deriving its clinical impact.

\section{REFERENCES}

Nance JW Jr, Crane GM, Halushka MK, Fishman EK, Zimmerman SL. Myocardial calcifications: pathophysiology, etiologies, differential diagnoses, and imaging findings. J Cardiovasc Comput Tomogr. 2015;9(1):5867.

Marciniak A, Marciniak M, Chiribiri A, et al. Multimodality imaging of extensive caseating intramyocardial calcification secondary to lymphoma. Circ Cardiovasc Imaging. 2015;8(4):e002750.

\section{FIGURE LEGEND}

Figure 1: A. Chest X-Ray showing a diffuse hypodense lobulated area in the left ventricle

Figure 1: B, Video 1. 2D-Echocardiogram revealing diffuse aortic and mitral calcifications with mild stenosis, marked septum and antero-lateral asymmetric hypertrophy, with extensive antero-lateral calcifications.

Figure 1: C, C1. CT-VRT reconstruction of the left ventricle, respectively axial view and focus on the coronal view.

Figure 1: C2, Video 2. Non-contrast CT MPR-axial view presents widespread calcifications in the left ventricular wall extended to the mitral-aortic annulus and both coronary arteries.

Figure 2: D, D1, Video 3. CMR SSFP-cine 4-chambers and short-axis views respectively showing lateral and antero-lateral wall thickening with areas of intramyocardial low signal, surrounded by normal 
myocardium.

Figure 2: E, E1. CMR T1weighted-spin-echo 4-chambers and short-axis view respectively show thickening of the lateral and antero-lateral wall with areas of low-signal.

Figure 2: F. CMR STIR 4-chambers showing lateral thickening with areas of low-signal.

Figure 2: G. CMR T1-native-mapping demonstrate diffuse septum and lateral fibrosis with low-signal in correspondence of the calcifications in the lateral wall (grey-light blue areas).

Figure 2: H, H1. CMR PSIR-sequences in 4-chamber view and short-axis view respectively. After contrastgadolinium injection, intramyocardial hypersignal (gadolinium enhancement) is shown in the antero-lateral wall.
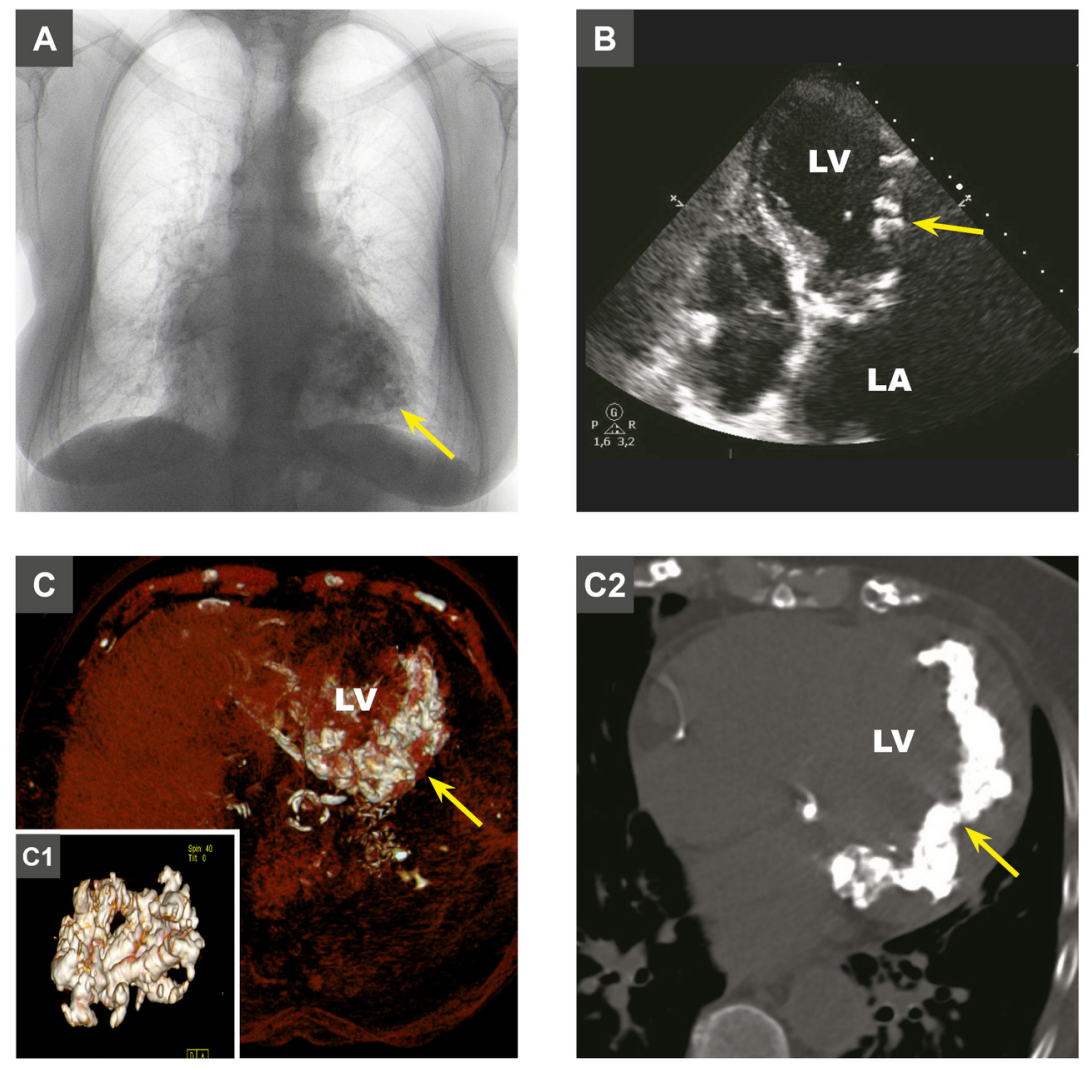

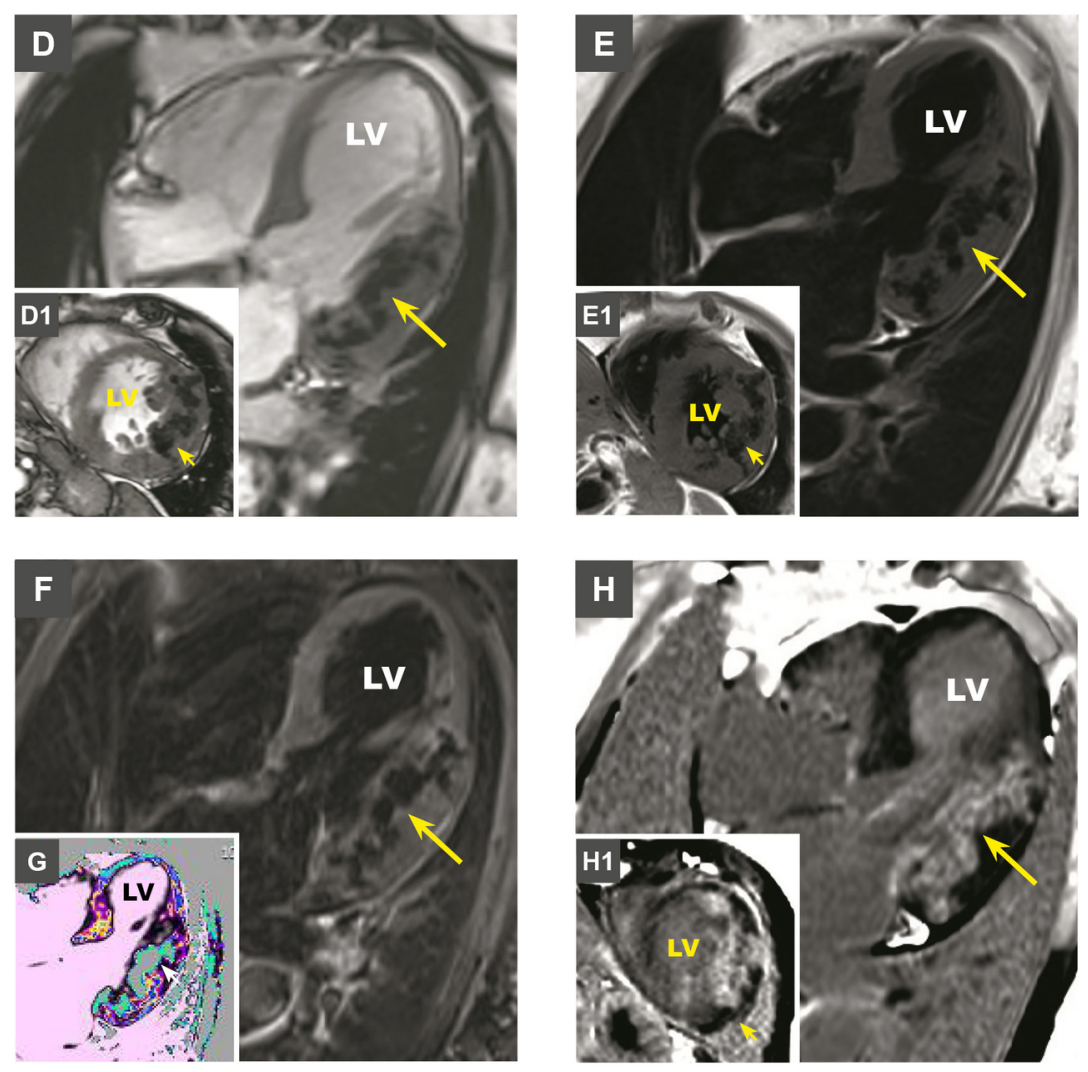